\title{
Attenuation of Pulmonary Neuroendocrine Differentiation in Mice Lacking Clara Cell Secretory Protein
}

Cesar M. Castro, Yongping Yang, Zhongjian Zhang, and R. Ilona Linnoila

Medicine Branch (CMC, YY, RIL), National Cancer Institute, National Institutes of Health, Rockville, Maryland, and Heritable Disorders Branch (ZZ), National Institute of Child Health and Human Development, National Institutes of Health, Bethesda, Maryland

SUMMARY: During development and injury, pulmonary neuroendocrine (NE) cells may transiently express Clara cell 10 kD protein (CC10), a major product of the nonciliated progenitor cells for normal and neoplastic airway epithelia suggesting a close relationship between the cells. To assess the role of CC10 during NE differentiation, we studied CC10-deficient mouse lungs by immunohistochemistry and digital imaging. The knockout model revealed a lack of the disrupted gene product in the lung. Because NE cells, which occur as solitary cells or in neuroepithelial bodies (NEBS), comprise less than $1 \%$ of airway epithelia, we counted foci positive for each of the three NE markers-synaptophysin, calcitonin gene-related peptide (CGRP), and protein gene product (PGP) 9.5-and developed a method to analyze numerous airways in serial sections. Digitized images of slides were segmented with Photoshop imaging software. The length of airway epithelium and total section areas were then measured using MetaMorph image analysis software. A comparable range of NE foci was observed regardless of CC10 expression patterns with all three markers, suggesting that CC10 is not critical for NE ontogenesis. However, discrimination according to size revealed that wild-type lungs harbored $30 \%$ to $40 \%$ greater synaptophysin- and CGRP-containing NEBs relative to CC10 deficient lungs. We posit that an attenuation of pulmonary NE differentiation afflicts the CC10-deficient state. Our imaging application greatly facilitates the acquisition and analysis of complex structures such as the lung and promises to be a widely applicable technique for assessments of tissue sections. (Lab Invest 2000, 80:1533-1540).

$P$ ulmonary neuroendocrine (NE) cells comprise a rare and well-differentiated subpopulation of airway epithelial cells characterized by the presence of dense-core granules (Linnoila, 1994). They occur as solitary cells or may form small organoid clusters called neuroepithelial bodies (NEBs). Through their hormone production, NE cells have been implicated in epithelial cell growth, branching morphogenesis, and carcinogenesis including small cell lung cancer, the most common and fatal of human NE cancers (Aguayo et al, 1994; King et al, 1995; Linnoila and Aisner, 1995). Pulmonary NE cells are ultimately derived from a common multipotent stem cell in the airway epithelium which bears a close relationship with nonciliated secretory or Clara cells. Findings suggest that hyperplastic pulmonary NE cells, which are associated with epithelial damage and carcinogenesis, may also express Clara cell 10 kD secretory protein (CC10) (Khoor et al, 1996; Reynolds et al, 2000). This prompted us to further study the relationship of CC10 with pulmonary NE cells.

CC10, composing seven percent of the total protein in normal bronchioloalveolar lavage fluid, is the major

Received June 23, 2000.

Address reprint requests to: Dr. R. Ilona Linnoila, National Cancer Institute, 9610 Medical Center Drive, Suite 300, Rockville, MD 20850. Fax: 301402 4422; E-mail: il17 h@nih.gov protein product of the nonciliated secretory or Clara cells that are precursors for neoplastic and nonneoplastic peripheral airway epithelia (Van Winkle et al, 1996). CC10 protein and mRNA-containing cells have been detected throughout the human tracheobronchial tree with their greatest abundance in the distal airways (Broers et al, 1992; Jensen et al, 1994; Linnoila et al, 1992). Despite a myriad of biological activities ascribed to $\mathrm{CC} 10$, its specific in vivo role in lung biology remains unknown, although studies do intimate immuno-modulatory and anti-inflammatory roles (Mukherjee et al, 1999). Specifically, the inhibition of phospholipase $A_{2}$ activity, the rate-limiting enzyme in the production of arachidonic acid, has been detected in vitro. This concurs with a biologically plausible mechanism preventing lungs from a grossly exaggerated, and thus deleterious, response to the multitude of environmental toxins and noxious agents. Moreover, CC10 bronchioalveolar lavage levels are decreased in fluids from patients suffering from lung diseases or asthma (Shijubo et al, 1999). Our previous studies suggest that CC10 expression affects cancer progression (Szabo et al, 1998; Zhang et al, 1999). Therefore, assessing differentiation pathways and abnormalities of the airway epithelia of mice bearing a disrupted CC10 gene may offer us further insights into the functions of the CC10 protein itself.

The aim of the current study was to determine whether, through the use of knockout mice, pulmo- 


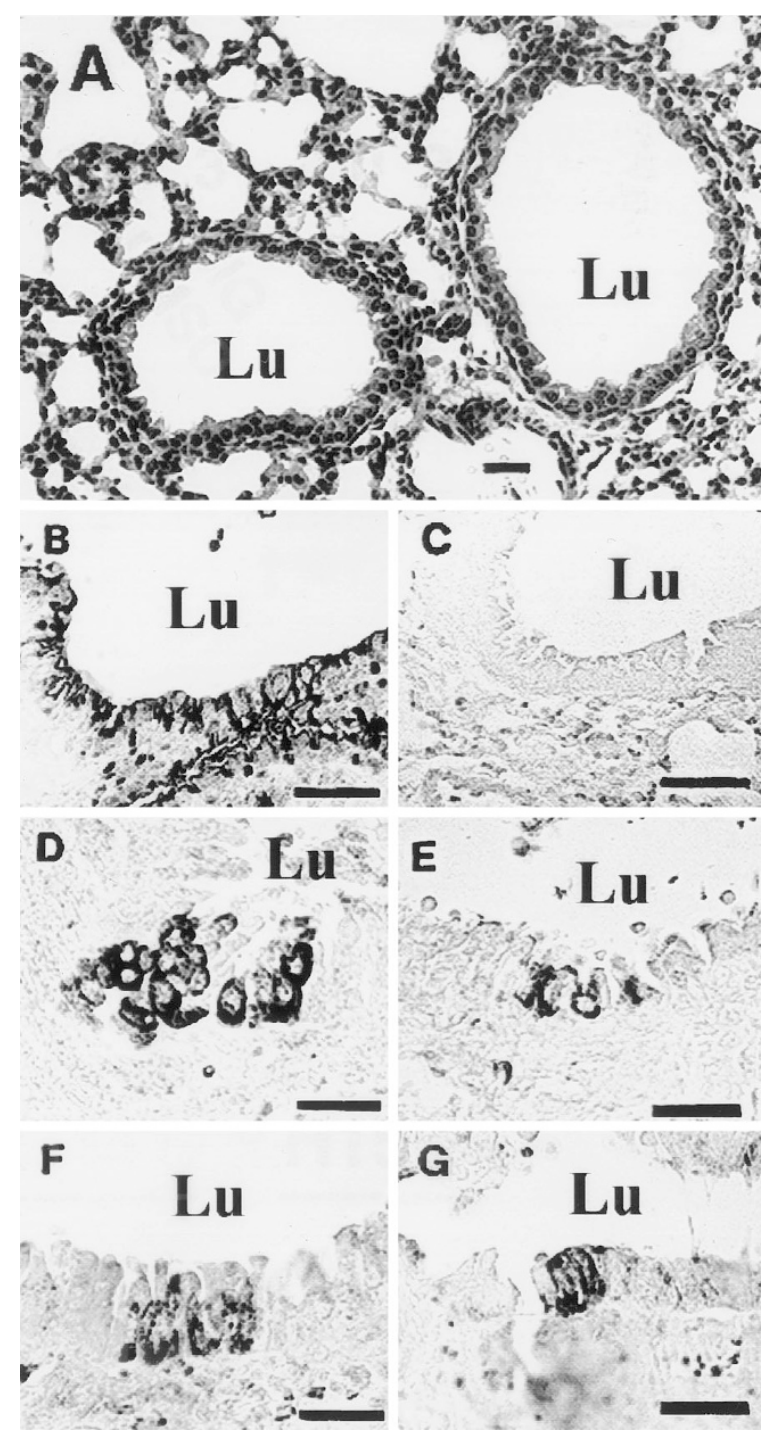

Figure 1.

Photomicrographs of wild-type and knockout lungs. A hematoxylin and eosin section demonstrating two well-developed and intact pulmonary bronchioles from a CC10-deficient mouse is shown in $A$. Immunohistochemical staining with anti-CC10 antibody shows expression in the majority of airway epithelial cells of the wild-type mouse lung $(B)$, whereas the CC10 knockout mouse airways lack immunoreactivity $(C)$. Mouse lung staining for CGRP, one of our NE markers, revealed that NE foci in the airways were less differentiated and smaller in size in the CC10-deficient mouse lung $(E)$ compared with its CC10-positive counterpart $(D)$, characterized by abundant, dark, cytoplasmic immunoprecipitate. This pattern of NE differentiation was confirmed by immunohistochemical staining with anti-synaptophysin antibody, another NE marker, as seen in wild-type $(F)$ and knockout $(G)$ specimens. ( $L u=$ bronchiolar lumen; $b a r=25 \mu \mathrm{m}$ ).

nary NE cell differentiation was affected by the removal of $\mathrm{CC} 10$ expression, along with its pluripotential influences, from the epithelium. Detection of NE differentiation necessitates specialized techniques including immunohistochemistry. Moreover, the relative rarity of NE cells, coupled with the vastness and complexity of the lung, prompted us to develop an approach that would steer away from subjective descriptions and inaugurate more objective quantitative methods for analyzing large numbers of intrapulmonary airways. Advances in computer hardware and

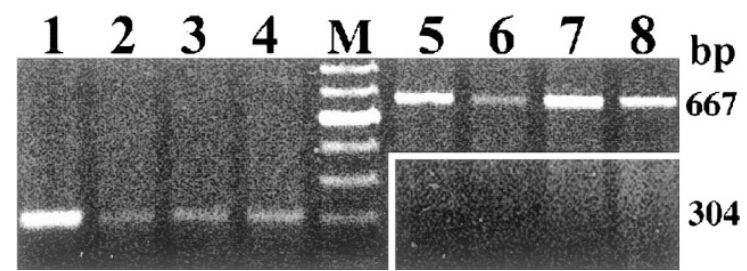

Figure 2.

PCR analysis of genotype. Representative PCR analyses of genomic DNA from mouse lung sections. The genotypes and their corresponding PCR products are as follows: 304-bp bands represent the genotype for CC10 wild-type mice $(C C 10+/+)$; $667-$ bp bands represent the genotype for CC10-deficient mice (CC10-/-). Lanes 1, 2, 3, 4 are derived from CC10 wild-type mice with same strain background. Lanes 5, 6, 7, 8 are from CC10 knockout mice. The white box merely highlights the absence of the wild-type, confirming $304 \mathrm{bp}$.

software technologies enabled us to devise a novel procedure for segmenting and measuring lung airway sections with a $35-\mathrm{mm}$ scanner and available commercial software.

\section{Results}

\section{Histology and Genotyping}

We ascertained CC10 positiveness or deficiency in mice by immunostaining the lung sections with antibodies raised against CC10 (Fig. 1, B and C). We then confirmed the CC10 immunohistochemical data by genotyping each specimen via DNA polymerase chain reaction. Using genotype-specific primers, the samples from knockout mice revealed the presence of a 667-base pair (bp) fragment, whereas wild-type animals were characterized by a 304-bp fragment (Fig. 2).

Lung maturation was determined by examining its tissue morphology and by assessing the expression patterns of genes intimately involved in its epithelial and NE differentiation. Light microscopy-based evaluation of hematoxylin and eosin (H\&E) sections revealed that $\mathrm{CC} 10$-deficient mice presented welldeveloped, unremarkable, and intact lung structures (Fig. 1A). No evidence of either hyperplasia or inflammation existed. Expression of CC10 was completely absent in knockout mice (Fig. 1, B and C). Immunoreactivity for the NE differentiation markers-calcitonin gene-related peptide (CGRP), synaptophysin, and protein gene product (PGP) 9.5-was seen in scattered epithelial cells and nerve fibers along the conducting airways in both CC10-deficient and wild-type animals (Fig. 1, D to G). CGRP is the major hormonal product of NE cells in rodent lungs, and synaptophysin represents a general marker for NE cells in the diffuse NE system. Likewise, PGP9.5 is widely expressed in neuronal tissues including pulmonary NE cells (Linnoila, 1994). To further compare the degree of pulmonary NE cell differentiation in both animal groups, we counted the number of CGRP-, synaptophysin-, and PGP9.5-positive foci (composed of solitary cells and NEBs), measured cross sections of the NEBs $\left(\mu \mathrm{m}^{2}\right)$, and determined the lengths of airways $(\mathrm{cm})$ using the steps illustrated in Figure 3. In addition, we determined the areas of tissue sections $\left(\mathrm{mm}^{2}\right)$. 


\section{Image Acquisition and Pre-Processing}

The purpose of the first step (Fig. 3) was to efficiently acquire a high-resolution image of an entire mouse lung section and optically outline the airways. To achieve this, H\&E-stained glass slides were digitized using a scanner. We used Photoshop imaging software (Adobe Systems, Mountain View, California) to facilitate acquisition with minimal background cast. The resulting 9600 dots-per-inch (dpi) color positive scans of mouse lungs were subsequently separated into individual lobes (Fig. 4A).

The digitized color image is the resulting superimposition of its red (R), blue (B), and green (G) channels, storing red, blue, and green information, respectively. An autoleveling step of these channels yielded our precursor image for segmentation. This step defines the lightest and darkest pixels from each RGB channel as white and black and then, in proportionate fashion, redistributes the intermediate pixel values. Photoshop's myriad algorithms allow for facile mathematical manipulation of images. Calculating the sum (via the Add function) of the autoleveled image's $R$ and $B$ channels yielded an RB image. This calculation performs algorithms on the corresponding pixels of the two channels and combines them into a single channel. The rationale behind the summation of the image's red and blue channels lies in the characteristic purplish tint exhibited by the airway lining cells in the natural setting. By digitally elucidating this feature, the epithelium becomes more pronounced. This segmentation step exclusively highlighted the airway epithelium and omitted structures not of interest to us, such as blood vessels (Fig. 4B). Similar results were obtained using immunostained slides counterstained by Light Green (Sigma, St. Louis, Missouri) using autoleveling. The tinctorial properties of green eliminated the need to summate separate $R$ and $B$ channel images.

\section{Airway Classification}

The lung slides revealed several generations of airways ranging from large bronchi to terminal bronchioli.

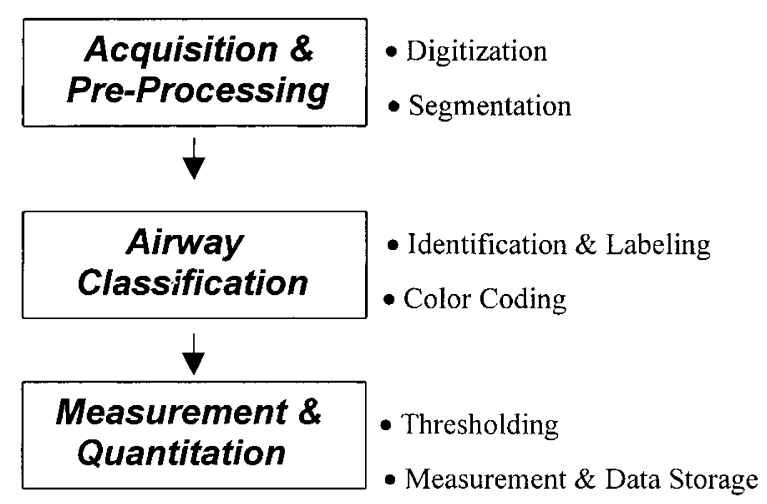

Figure 3.

Schematic view of airway analysis. Our imaging technique included the requisite acquisition and pre-processing, airway classification, and measurement and quantitation processes for effective analysis. The unique digitization and segmentation techniques employed facilitated the subsequent steps.
The purpose of the next step (Fig. 3) was to classify these structures. Color coding of airways was performed by filling the lumen with the color depicted in our airway class key (Fig. 5A). To perform reliable MetaMorph (Universal Imaging, West Chester, Pennsylvania) software quantitation, open airways (terminal bronchioles joining alveolar ducts and parenchyma) required "blocking" to preclude a color and measurement runoff in later steps. This involved the placement of a block line from the airway epithelial terminus to the terminus at the bronchioloalveolar junction (Fig. 5B). Line distances would later be measured and subsequently subtracted from the perimeters of thresholded airways. Images were stored as tagged image file format (TIFF) files in preparation for measurement.

\section{Measurement and Quantitation}

Adobe Photoshop and MetaMorph imaging software linked to Microsoft Excel (Microsoft, Redmond, Washington) via Dynamic Data Exchange served as the software components of our laboratory's imaging analysis system. To each airway class, distinguished by its assigned color, we applied the software's image enhancement technique or thresholding. In other words, the operator selected one color-coded structure from the airway class of interest. That color would then be added to the threshold level. Hence, all airway structures of that particular class would now be thresholded simultaneously. MetaMorph's Integrated Morphometry Analysis program equipped us with a large variety of available measurement parameters. For our purposes, airway length data were obtained by measuring the perimeters of thresholded airway lumens. In the case of terminal airways that opened to the alveoli, the operator also measured the length of the bars blocking open airways and subtracted them from the total perimeter. Data was sorted and tabulated in a manner consonant with our interests (Table $1)$.

In a pilot experiment of 10 animals, a small but statistically significant difference in total airway lengths and numbers was evident between the CC10positive and -negative groups. Such a difference was manifest in the bronchiolar lengths as well (Table 1). Because subsequent mouse specimens contained incomplete lungs, one part having been frozen for further studies, we determined the total length of only the airways that were derived from similar specimens (Table 2). Section areas of each slide were measured from scanned images with MetaMorph's Integrated Morphometry Analysis program after applying inclusive segmentation. Total areas examined for each marker in both animal groups were similar, ranging from $58 \pm 19$ to $66 \pm 21 \mathrm{~mm}^{2}$ (mean $\pm \mathrm{SD}$ ), containing from $97 \pm 19$ to $119 \pm 37$ airways in each (Table 2 ).

\section{Analysis of Neuroendocrine Cells}

After establishing that the serial sections in each group were comparable, the total number of NE foci 

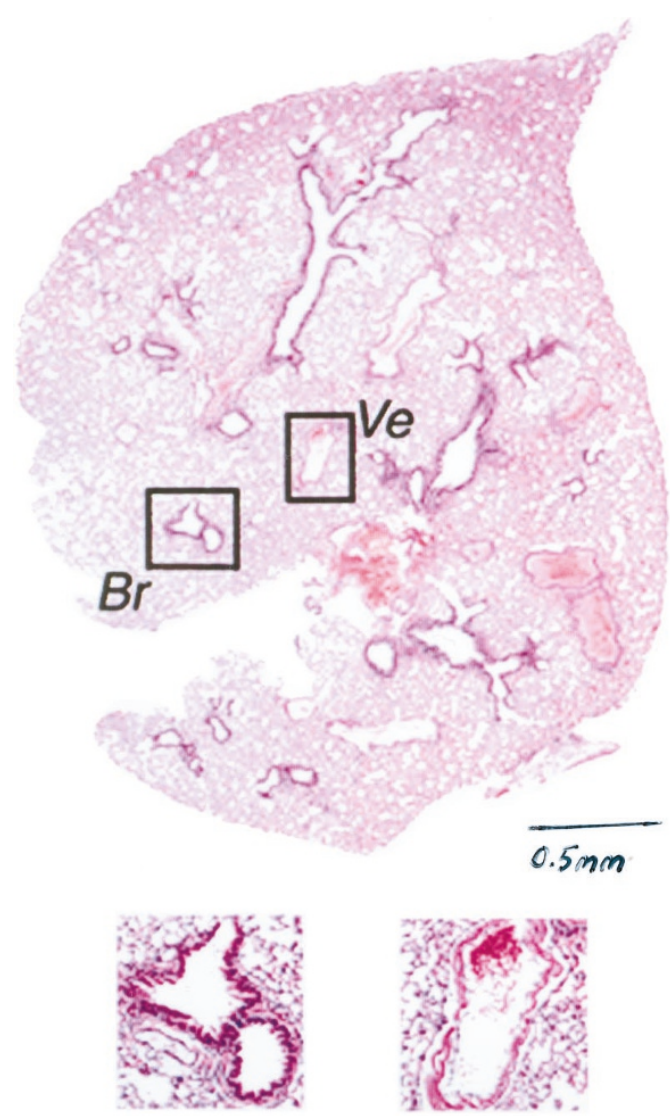

$\mathrm{Br}$

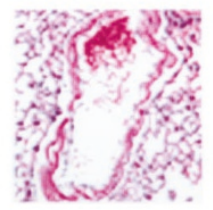

Ve

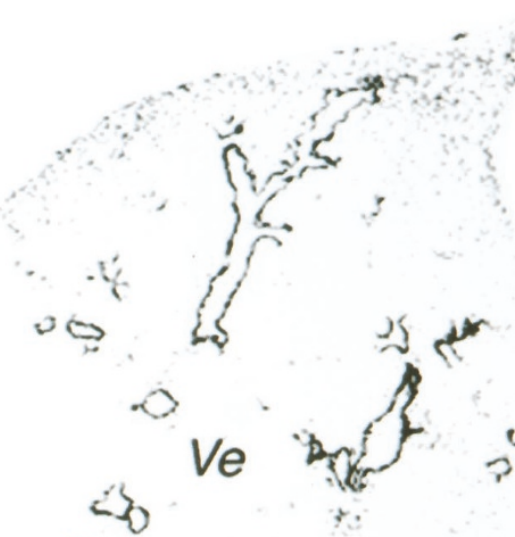

$\mathrm{Br}$
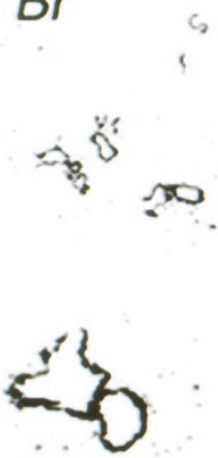

$\mathrm{Br}$

Figure 4.

Digitized image with airway segmentation. $A$, Scanning at 9600 dpi yielded a whole-mount image amenable to quick separation into lobes. Detailed views of a bronchiole $(B r)$ and a vessel $(V e)$ from the true H\&E are shown. $B$, Photoshop's myriad algorithms enabled multiple manipulations, which in our case included the summation of the image's red and blue channels. The bronchiole $(B r)$ remained visible whereas the blood vessel (Ve) and alveoli were diffused out, consequently elucidating epithelium and facilitating its coding and thresholding later on.

consisting of solitary cells or NEBs immunoreactive for CGRP, synaptophysin, or PGP9.5 in serial sections were counted with a microscope (Table 2). Each lung section had 17 to $22 \mathrm{NE}$ foci (mean). To compensate for possible discrepancies among airway numbers, total lengths of airways, or section areas among slides from various animals, we related the counts of NE foci to these parameters as measured in adjacent H\&E slides (CGRP) or actual immunoslides (synaptophysin and PGP9.5). The ratios of NE foci per airway number, per airway length, and per section area for each marker were comparable between the wild-type animals that expressed $\mathrm{CC} 10$ and knockout animals that were negative for CC10 (Table 2). There was no correlation with age (data not shown).

The majority $(57 \%$ to $66 \%$, depending on the marker) of NE foci were NEBs, which are collections of three or more NE cells growing in organoid-type clusters that may be innervated (Linnoila, 1994). Our visual impression that NEBs in wild-type animals were better developed than those in knockout animals prompted us to further characterize the degree of $\mathrm{NE}$ differentiation. Digital images of the NEBs by each marker were collected via microscope using a $\times 20$ objective, and the immunoreactive NEB areas were measured using MetaMorph's Integrated Morphometry Program. The mean area of staining for each NEB containing CGRP, as well as synaptophysin in the CC10-expressing animals, was $31 \%$ to $39 \%$ smaller in knockout animals $\left(252 \mu \mathrm{m}^{2}\right.$ vs $153 \mu \mathrm{m}^{2}, p=0.04$; and $337 \mu \mathrm{m}^{2}$ vs $232 \mu \mathrm{m}^{2}, p=0.038$, respectively; Table 2). In contrast, PGP9.5-containing NEBs were similar in size in wild-type and CC10 knockout animals ( $235 \pm$ $105 \mu \mathrm{m}^{2}$ vs. $\left.220 \pm 90 \mu \mathrm{m}^{2}\right)$. The size differences are illustrated in Figure 1, D to G, and in Figure 6, which concerns the area of NEBs as the function of animal age. The decrease in the NEB size of knockout animals was most pronounced in the 2-month-old group with CGRP.

\section{Discussion}

During epithelial injury, CC10 is coexpressed with an increasing number of pulmonary neuroendocrine cells (Reynolds et al, 2000; Stripp et al, 1995). Other studies have detected a close spatial relationship between CC10 and NE foci insofar as to conclude an interaction between them during lung development (Khoor et al, 1996). To further assess the in vivo role of CC10 during carcinogenesis and NE differentiation, we stud- 


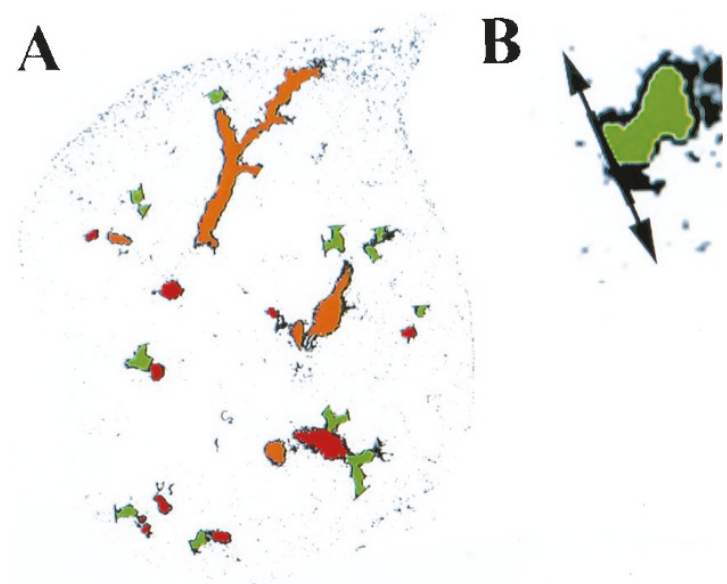

Figure 5.

Airway thresholding. A, The segmented image's airway classes were color coded according to the following: bronchioli with muscle, red; bronchioli, orange; terminal bronchiole, green. We employed the original H\&E image (Fig $4 \mathrm{~A})$ as a visual aid for classifying the pre-processed or segmented airways (Fig. 4B) according to size and histology. Bronchi (code 1) were lined with pseudostratified columnar ciliated epithelium. The lamina propria was surrounded by a layer of smooth muscle, and cartilage plates were visible. Bronchioli (code 2, orange) were about $1 \mathrm{~mm}$ in diameter. Mucosal folds were prominent, and the epithelium consisted of low columnar cells encompassed at times by a muscle layer (bronchioli with muscle, code 3 , red). Terminal bronchioles (code 4, green) were open-airway structures containing mainly cuboidal ciliated and nonciliated cells. First, each structure was assigned a number (codes 1-4) according to its class. Each color and, hence, airway class was subsequently thresholded and their perimeters measured. $B$, In the case of terminal bronchioles, which by definition are open structures, manual blockage was needed to preclude runoff. The lengths of the bars were later subtracted from the perimeters.

ied the lungs of CC10-deficient mice. We observed a range of pulmonary NE cells in mice, regardless of their genotype. The presence of these cells in CC10 knockout mice suggests that this protein may not be critical for normal NE ontogenesis. In mice lacking CC10 expression, the relative number of airway foci expressing NE markers was comparable to their normal counterparts. Yet, the appearance of lighter staining intensity in the knockout mice, coupled with reduction in staining areas and smaller NEBs, which was statistically significant, points to a developmental attenuation of pulmonary NE differentiation in the absence of CC10. The fact that two markers gave similar results suggests that the deficiency affects the cellular differentiation rather than the expression of an individual gene.

The relationship between Clara cells and pulmonary NEs is not disparate. Pulmonary NEs have been postulated to derive from pulmonary endoderm and not neural crest (Hoyt et al, 1990). Wuenschell et al (1996) observed that CGRP, CC10, and surfactant protein A immunohistochemically were coexpressed in most cells of the distal lung epithelium during a certain period of fetal development, later to mature into their distinct lineages. Moreover, the observation that CC10-staining cells tend to form "crowns" over NE cells is suggestive of an intimate dynamic. We posit that the lack of $\mathrm{CC} 10$ during lung development disrupts any early interactional effects between $\mathrm{CC} 10$ and CGRP in the epithelial lining, resulting in diminished growth of NE foci. The pattern of reduced growth may also be associated with synaptophysincontaining NEBs. NE cell ontogenesis, however, remains unaffected, as evidenced by the comparable relative incidences of NE foci in wild-type and CC10deficient mice. The fact that there was no change in the size of PGP9.5 potentially indicates a divergent relationship with CC10. Interestingly, the expression of PGP9.5 has recently been associated also with non-NE phenotype during lung carcinogenesis (Hibi et al, 1999). These results suggest that PGP9.5, which is a ubiquitin hydrolase, may be independent of $\mathrm{NE}$ differentiation. A possible direction arising from our results would involve evaluating the size of NE foci in mice with overexpression of $\mathrm{CC} 10$ and ascertaining whether the larger NE foci confer a growth advantage.

Light microscopic evaluation revealed normal and intact pulmonary structures in the CC10 knockout and wild-type mice. There were small, but statistically significant, differences in the airway lengths between wild-type and knockout mice. These differences were clouded by the disparity in airway numbers and the small number of animals with intact lungs and may reflect variable tissue positioning before sectioning. Given the collection of lung tissues we had, the more informative interpretation was to relate such differences to the NE foci numbers as we did in the current paper. Our scanning and image segmentation technique successfully tackled a previously formidable and laborious task. The sheer quantity (over 8000 ) of sectioned airways to be reviewed had compelled us to seek an efficient alternative. Our imaging protocol minimizes interactivity between operator and images. The color-coding and subsequent one-step thresholding of each airway class proved more reproducible and faster than previous manual thresholding attempts of each airway individually (data not shown). The digitization of the glass slide section offers sufficient resolution to discern airway epithelia from vessel endothelia and alveoli. Our prior methodologies involved the laborious image capture of tissue section quadrants via a photomicroscope and subsequent analysis with imaging software. Scanning the actual glass slide rapidly yields whole section images and maps of the lobe architecture. This precludes any subsampling steps arising from the limitations of a microscope's smaller field of view. The approach also greatly facilitated the evaluation the lung area examined in each serial section. The fast and reliable determination of tissue area has immediate further applications for molecular pathology where the yield of DNA or RNA is directly proportional to the amount of tissue. For instance, we foresee our scanning and measuring techniques as viable quantitative complements to the laser capture microdissection procedure.

Moreover, scanned images may be printed at 300 to 600 dpi for navigational, teaching, and recordkeeping purposes. Printing scanned, serially cut sections onto transparencies and stacking them can also offer quick albeit rudimentary three dimensional information. Scanning also provided uniform illumination not easily 
Table 1. The Length and Number of Intrapulmonary Airways According to Class

\begin{tabular}{lccccc}
\hline Animal ID & $\begin{array}{c}\text { CC10 } \\
\text { Expression }\end{array}$ & $\begin{array}{c}\text { Bronchioles }^{a} \\
\text { with Muscle } \\
\text { Length }(\mathrm{n})^{b}\end{array}$ & $\begin{array}{c}\text { Bronchioles } \\
\text { Length }(\mathrm{n})\end{array}$ & $\begin{array}{c}\text { Terminal } \\
\text { Bronchioles } \\
\text { Length (n) }\end{array}$ & Total Length (n) \\
\hline 289 & Positive & $8.8(2)$ & $47.2(89)$ & $39.2(67)$ & $95.2(158)$ \\
290 & Positive & $11.6(6)$ & $48.9(86)$ & $22.5(76)$ & $83.0(168)$ \\
291 & Positive & $39.6(25)$ & $53.9(67)$ & $37.3(53)$ & $130.8(145)$ \\
293 & Positive & $37.5(35)$ & $36.4(75)$ & $45.6(73)$ & $119.6(183)$ \\
294 & Positive & $34.0(25)$ & $52.0(47)$ & $47.3(60)$ & $133.3(132)$ \\
$\operatorname{Mean}^{c}(5)$ & Positive & $26.3 \pm 15(19)$ & $48.7 \pm 7^{d}(71)$ & $38.4 \pm 10(65)$ & $112.4 \pm 22^{e}(157)^{f}$ \\
13542 & Negative & $3.3(1)$ & $32.1(55)$ & $21.4(39)$ & $56.8(95)$ \\
13543 & Negative & $16.2(4)$ & $30.2(68)$ & $18.3(60)$ & $64.7(132)$ \\
13006 & Negative & $14.1(2)$ & $34.6(58)$ & $29.9(68)$ & $78.6(128)$ \\
13007 & Negative & $23.4(6)$ & $31.4(59)$ & $35.9(55)$ & $90.7(120)$ \\
92063 & Negative & $45.6(7)$ & $33.8(64)$ & $30.1(70)$ & $109.5(141)$ \\
$\operatorname{Mean}^{c}(5)$ & Negative & $20.5 \pm 16(4)$ & $32.4 \pm 2^{d}(60)$ & $27.1 \pm 7(58)$ & $80.1 \pm 21^{e}(123)^{f}$ \\
\hline
\end{tabular}

${ }^{a}$ Intrapulmonary airways were classified into 4 groups and in accordance with published morphological criteria. Because only one animal in each category had a bronchus included in the section, we omitted that category.

${ }^{b}$ The lengths were measured by image analysis and expressed as total lengths in millimeters for each airway type. The number of airways is included in parenthesis.

${ }^{c}$ For each airway class, average lengths and standard deviations were obtained from CC10-positive or -negative expressing mice.

${ }^{d}$ There was a statistically significant difference in the length of bronchioles between the groups ( $p=0.001$; Mann-Whitney $\mathrm{U}$ test).

${ }^{e}$ There was a statistically significant difference in the total lengths of airways between the groups ( $p=0.046$; Mann-Whitney U).

${ }^{f}$ There was a statistically significant difference in total number of airways between the groups ( $p=0.021$; Mann-Whitney $\left.\mathrm{U}\right)$.

Table 2. Pulmonary NE Differentiation in Wild-Type and CC10 Knockout Mice (mean \pm SD)

\begin{tabular}{|c|c|c|c|c|c|c|c|c|}
\hline Genotype (n) & Marker & $\begin{array}{l}\text { Number of } \\
\text { Airways }\end{array}$ & $\begin{array}{c}\text { Area of Lung } \\
\text { Section }\left(\mathrm{mm}^{2}\right)\end{array}$ & $\begin{array}{c}\text { Length of } \\
\text { Airways }(\mathrm{cm})\end{array}$ & $\begin{array}{l}\text { NE Foci per } \\
\text { Animal }\end{array}$ & $\begin{array}{l}\text { NE Foci per } \\
\text { Airway }\end{array}$ & $\begin{array}{l}\text { NE Foci/mm of } \\
\text { Airway }\end{array}$ & $\begin{array}{c}\text { Area of NEBa } \\
\left(\mu \mathrm{m}^{2}\right)\end{array}$ \\
\hline \multirow[t]{2}{*}{ Wildtype (15) } & CGRP & $119 \pm 37$ & $58 \pm 19$ & $5.2 \pm 2.5$ & $19 \pm 10$ & $0.19 \pm 0.13$ & $46 \pm 23$ & $252 \pm 115$ \\
\hline & SYN & $102 \pm 36$ & $58 \pm 20$ & $5.3 \pm 2.8$ & $17 \pm 11$ & $0.19 \pm 0.11$ & $35 \pm 13$ & $337 \pm 140$ \\
\hline \multirow{2}{*}{ Knockout (11) } & CGRP & $106 \pm 44$ & $66 \pm 21$ & $6.4 \pm 3.2$ & $17 \pm 10$ & $0.16 \pm 0.08$ & $38 \pm 30$ & $153 \pm 39$ \\
\hline & SYN & $97 \pm 19$ & $62 \pm 19$ & $5.8 \pm 2.2$ & $22 \pm 5$ & $0.27 \pm 0.17$ & $42 \pm 25$ & $232 \pm 45$ \\
\hline
\end{tabular}

$\mathrm{NE}$, neuroendocrine; CC10, Clara cell $10 \mathrm{kD}$ protein; CGRP, calcitonin gene-related peptide; SYN, synaptophysin.

${ }^{a}$ There was a statistically significant difference in the staining areas of NEBs between the groups in the CGRP analysis $(p=0.013)$ and SYN analysis $(p=0.032)$ (Mann-Whitney U test).

achieved by charged couple device cameras attached to microscopes using low-power objectives. The sum of the scanned images' red and blue channels exploited the H\&E staining patterns of epithelia. The advantage of our method lies in the minimized interactivity between operator and image during the most crucial and problematic step of the analysis, ie, segmentation. Depending on the tissue type and staining characteristics, the researcher may easily tailor the calculation algorithms according to his or her needs. Nevertheless, when the research entails numerous tissue sections to be analyzed, efficiency and reproducibility become key concerns.

We have demonstrated the feasibility of a rigorous analytical procedure for segmenting and measuring over $1.5 \mathrm{~m}$ of mouse airways using a $35 \mathrm{~mm}$ slide scanner at $9600 \mathrm{dpi}$. Moreover, we have begun to implement digital scanning in the comparison of staining relationships and the conservation of the spatial relationship between the antigen of interest and tissue morphology (Elia et al, 2000; Ruifrok, 1996). With a slight augmentation in hardware scanning technology, we endeavor to facilitate rapid detection, via immunohistochemistry, of relative changes in intracellular pep- tide content by linking the supraoptimal dilution technique with our imaging application (Mize, 1994; Springall and Polak, 1997).

\section{Materials and Methods}

\section{Specimens and Immunohistochemistry}

CC10 (also called uteroglobin) knockout mice were generated via gene-targeting in embryonic stem cells as previously described (Zhang et al, 1997). Animals were serially killed at 2 months, 7-10 months, and 16 months of age. Their lungs were gently inflated with 1 $\mathrm{ml}$ tracheal infusion and fixed overnight using 10\% formalin and embedded in paraffin. Sections $(5-\mu \mathrm{m}$ thick) on glass slides were H\&E stained and coverslipped. Serial sections of 11 knockout and 15 wildtype animals of comparable ages were deparaffinized and subsequently microwaved according to protocol to unmask antigens and increase the overall reaction sensitivity. The sections were then immunohistochemically stained via the avidin-biotinylated peroxidase method using the Vectastain ABC kit (Vector, Burlingame, California) with modification of vendor 


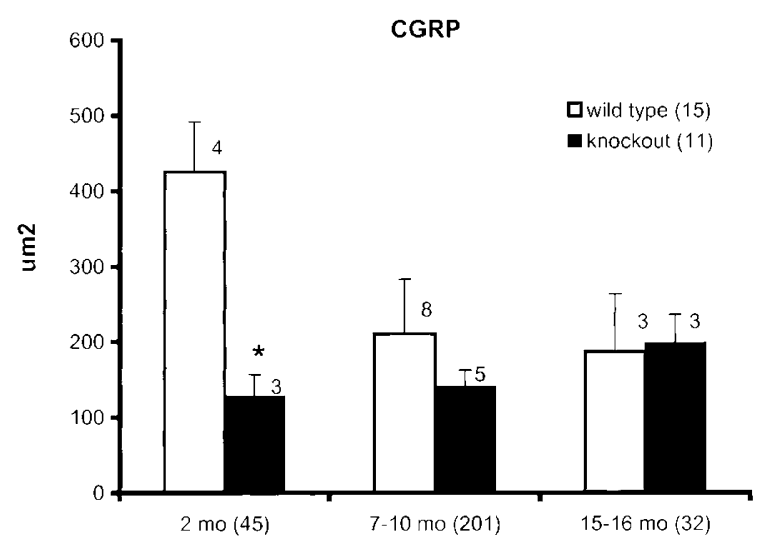

A

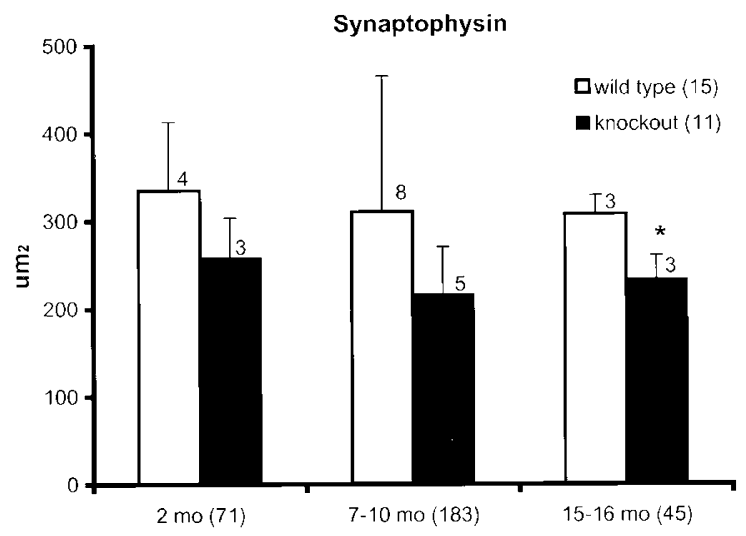

B

\section{Figure 6.}

The size of neuroepithelial bodies (NEBs) in CC10-deficient mice according to animal age. The barograph shows data from wild-type (open bars) and knockout (solid bars) mice with no CC10 expression following immunohistochemical staining for synaptophysin and CGRP. Animals represented three different age groups $\left(x\right.$ axis). The area of NEBs $\left(\mu \mathrm{m}^{2}\right)$ composed of three or more NE cells was measured using MetaMorph's Integrated Morphometry Program. The number of animals appears by the genotype. The number of NEBs measured is shown in parentheses by the age group. An asterisk denotes a statistically significant difference between the areas of wild-type and knockout animals' NEBs ( $p=0.017$ for synaptophysin and CGRP; MannWhitney U test).

directions (Linnoila et al, 1992). Pulmonary NE cell differentiation was assessed by incubating sections with rabbit polyclonal antibodies raised against synaptophysin (diluted 1:100) (Zymed, San Francisco, California), CGRP - the major peptide product of pulmonary NE cells in rodents-(diluted 1:3000) (Amersham, Uppsala, Sweden), and PGP9.5 (diluted 1:6000) (Biogenesis, Sandown, New Hampshire). Antibodies raised against CC10 (diluted 1:10,000) (a gift from Dr. Francesco DeMayo) and Urinary Protein -1 (UP-1)another antibody able to recognize $\mathrm{CC} 10$-(diluted 1:1000) (DAKO, Glostrup, Denmark) were employed to localize CC10 expression.

\section{DNA Extraction and Genotyping}

Lung tissue from $5-\mu \mathrm{m}$ paraffin sections was placed in an Eppendorf tube containing $100 \mu$ l of digestion buffer ( $2 \mu \mathrm{l}$ of $10 \mathrm{mg} / \mathrm{ml}$ proteinase $\mathrm{K} ; 20 \mathrm{~mm}$ Tris-HCL, pH 8.0; 5 mm EDTA; and 1\% Tween-20). The tube was incubated at $56^{\circ} \mathrm{C}$ for 4 hours, spun down briefly, and heated to $95^{\circ} \mathrm{C}$ for 8 minutes to inactivate proteinase $\mathrm{K}$.

We employed a DNA thermocycler (Perkin Elmer, Rockville, Maryland) to perform polymerase chain reaction (PCR). Five microliters of DNA from section samples were used for PCR amplification in a 50- $\mu$ l $P C R$ reaction mixture containing two units of Taq DNA polymerase (Gibco-BRL, Rockville, Maryland). A hotstart PCR was performed for 40 cycles of amplification under the conditions previously described (Zhang et al, 1997): $94^{\circ} \mathrm{C}$ for 1 minute, $58^{\circ} \mathrm{C}$ for 1.5 minutes, and $72^{\circ} \mathrm{C}$ for 1 minute with a final step at $72^{\circ} \mathrm{C}$ for 8 minutes. The PCR products were analyzed on a $2 \%$ agarose gel stained with ethidium bromide. The knockout (-/-) genotype was assessed by using a set of neo-specific primers, neo-L (5'- ATA CGC TTG ATC CGG CTA CCT GCC- 3') and neo-R (5'- CAT TTG CAC TGC CGG TAG AAC TCC -3'), yielding a 667-bp fragment. A 304-bp DNA fragment, generated with a set of uteroglobin (UG)-specific primers, mUG-L (5'ACA TCA TGA AGC TCA CAG GTA TCG-3') and mUG-R (5'-GTG TGC ACG GTT CAA GCT TGT AGT$\left.3^{\prime}\right)$, confirmed the wild-type genotype.

\section{Imaging Tools}

The hardware components of our system consisted of a Nikon LS-3510AF 35-mm slide scanner (9600 dpi), the Nikon Eclipse E400, equipped with Dage 3 Chip Color Camera and Microphot- FXA photomicroscopes (Image Systems Inc., Columbia, Maryland), and a Dell Optiplex GMT 5166 PC with a Pentium $166 \mathrm{MHz}$ processor and $64 \mathrm{MB}$ RAM. The imaging analysis software included MetaMorph Image Processing and Analysis System (Version 4.0; Universal Imaging Corporation, Westchester, Pennsylvania), which we used to measure quantitatively the airway perimeters as well as the areas of NE foci. Adobe Photoshop 5.5 (Adobe Systems, Mountain View, California) facilitated the classification, color coding, and scanning of mouse lung lobes and their structures. Microsoft Excel 97 (Microsoft, Redmond, Washington) served as our database program.

\section{Acknowledgements}

We thank Dr. Jerrold Ward of the Animal Sciences Branch, National Cancer Institute (Frederick, Maryland) for providing the mouse lung blocks. We also thank Ms. Tess Bunnag for her technical assistance in the development of the imaging application and Ms. Peg Prentice and Ms. Xu Naizhen for their tireless efforts in image acquisition and measurement.

\section{References}

Aguayo SM, Schuyler WE, Murtagh JJ Jr, and Roman J (1994). Regulation of lung branching morphogenesis by bombesin-like peptides and neutral endopeptidase. Am J Respir Cell Mol Biol 10:635-642. 
Broers JLV, Jensen SM, Travis WD, Pass H, Whitsett JA, Singh G, Katyal SL, Gazdar AF, Minna JD, and Linnoila RI (1992). Expression of surfactant associated protein-A and Clara cell 10 kilodalton mRNA in neoplastic and nonneoplastic human lung tissue as detected by in situ hybridization. Lab Invest 66:337-346.

Elia J, Aoki A, and Maldonado CA (2000). Response of bronchiolar Clara cells induced by a domestic insecticide. Analysis of CC10 kDa protein content. Histochem Cell Biol 113:125-133.

Hibi K, Westra WH, Borges M, Goodman S, Sidransky D, and Jen J (1999). PGP9.5 as a candidate tumor marker for non-small-cell lung cancer. Am J Pathol 155:711-715.

Hoyt RF, Jr., McNelly NA, and Sorokin SP (1990). Dynamics of neuroepithelial body (NEB) formation in developing hamster lung: Light microscopic autoradiography after $3 \mathrm{H}-$ thymidine labeling in vivo. Anat Rec 227:340-350.

Jensen SM, Jones JE, Pass H, Steinberg SM, and Linnoila RI (1994). Clara Cell $10 \mathrm{kDa}$ protein mRNA in normal and atypical regions of human respiratory epithelium. Int $\mathrm{J}$ Cancer 58:629-637.

Khoor A, Gray ME, Singh G, and Stahlman MT (1996). Ontogeny of Clara cell- specific protein and its mRNA: Their association with neuroepithelial bodies in human fetal lung and in bronchopulmonary dysplasia. J Histochem Cytochem 44:1429-1438.

King KA, Torday JS, and Sunday ME (1995). Bombesin and $\left[\mathrm{Leu}^{8}\right]$ phyllolitorin promote fetal mouse lung branching morphogenesis via a receptor-mediated mechanism. Proc Natl Acad Sci USA 92:4357-4361.

Linnoila RI and Aisner SC (1995). Pathology of lung cancer. In: Johnson B and Johnson D, editors. Lung cancer. New York: Wiley-Liss, 73-95.

Linnoila RI, Jensen SM, Mulshine JL, Eggleston JC, and Gazdar AF (1992). Peripheral airway cell marker expression in non-small cell lung carcinoma: Association with distinct clinicopathologic features. Am J Clin Pathol 97:233-243.

Linnoila RI (1994). Pulmonary endocrine cells in vivo and in vitro. In: Kaliner MA, Barnes PJ, Kunkel GHH, and Baraniuk JN, editors. Neuropeptides in respiratory medicine. New York: Marcel Dekker, 197-224.

Mize RR (1994). Quantitative image analysis for immunocytochemistry and in situ hybridization. J Neurosci Methods 54:219-237.
Mukherjee AB, Kundu GC, Mantile-Selvaggi G, Yuan C-J, Mandal AK, Chattopadhyay S, Zheng F, Pattabiraman N, and Zhang Z (1999). Uteroglobin: a novel cytokine? Cell Mol Life Sci 55:771-787.

Reynolds SD, Giangreco A, Power JH, and Stripp BR (2000). Neuroepithelial bodies of pulmonary airways serve as a reservoir of progenitor cells capable of epithelial regeneration. Am J Pathol 156:269-278.

Ruifrok AC (1996). Quantification of immunohistochemical staining by color thresholding and automated thresholding. Anal Quant Cytol Histol 19:107-113.

Shijubo N, Itoh Y, Yamaguchi T, Sugaya F, Hirasawa M, Yamada T, Kawai T, and Abe S (1999). Serum levels of Clara cell $10-\mathrm{kDa}$ protein are decreased in patients with asthma. Lung 177:45-52.

Springall DR and Polak JM (1997). Quantitative microscopial methods for the identification and localisation of nerves and neuroendocrine cell markers in mammalian lung. Microsc Res Tech 37:92-100.

Stripp BR, Maxson K, Meta R, and Singh G (1995). Plasticity of airway cell proliferation and gene expression after acute napthalene injury. Am J Physiol 13:L791-L799.

Szabo E, Goheer A, Witschi H, and Linnoila RI (1998). Overexpression of CC10 modifies neoplastic potential in lung cancer cells. Cell Growth Differ 9:475-485.

Van Winkle LS, Isaac JM, and Plopper CG (1996). Repair of naphthalene-injured microdissected airways in vitro. Am J Respir Cell Mol Biol 15:1-8.

Wuenschell CW, Sunday M, Singh G, Minoo P, Slavkin H, and Warburton D (1996). Embryonic mouse lung epithelial progenitor cells co-express immunohistochemical markers of diverse mature cell lineages. J Histochem Cytochem 44:113123.

Zhang Z, Kundu GC, Panda D, Mandal AK, Mantile-Selvaggi G, Peri A, Yuan CJ, and Mukherjee AB (1999). Loss of transformed phenotype in cancer cells by overexpression of the uteroglobin gene. Proc Natl Acad Sci USA 96:3963-3968.

Zhang Z, Kundu GC, Yuan C, Ward JM, Lee EJ, DeMayo F, Westphal H, and Mukherjee AB (1997). Severe fibronectindeposit renal glomerular disease in mice lacking uteroglobin. Science 276:1408-1412. 